\title{
Change in Postprandial Level of Remnant Cholesterol After a Daily Breakfast in Chinese Patients With Hypertension
}

Jin Xu

Second Xiangya Hospital

Peiliu Qu

Second Xiangya Hospital

Xiao Du

Second Xiangya Hospital

Qunyan Xiang

Second Xiangya Hospital

\section{Liling Guo}

Second Xiangya Hospital

Liyuan Zhu

Second Xiangya Hospital

Yangrong Tan

Second Xiangya Hospital

Yan Fu

Second Xiangya Hospital

Tie Wen

Second Xiangya Hospital

Ling Liu ( $\sim$ feliuling@csu.edu.cn )

Second Xiangya Hospital https://orcid.org/0000-0001-6979-701X

Research

Keywords: Hypertension, postprandial, remnant cholesterol, cut-off point

Posted Date: February 5th, 2021

DOI: https://doi.org/10.21203/rs.3.rs-181370/v1

License: (c) (1) This work is licensed under a Creative Commons Attribution 4.0 International License.

Read Full License 
1 Change in postprandial level of remnant cholesterol after a

Jin $\mathrm{Xu}^{1,2,3,4}$, Peiliu $\mathrm{Qu}^{1,2,3,4}$, Xiao $\mathrm{Du}^{1,2,3,4}$, Qunyan Xiang ${ }^{1,2,3,4}$, Liling Guo ${ }^{1,2,3,4}$, Liyuan

$\mathrm{Zhu}^{1,2,3,4}$, Yangrong Tan ${ }^{1,2,3,4}$, Yan $\mathrm{Fu}^{1,2,3,4}$, Tie Wen ${ }^{5,6}$, Ling Liu ${ }^{1,2,3,4 *}$

1. Department of Cardiovascular Medicine, the Second Xiangya Hospital, Central South University;

2. Research Institute of Blood Lipid and Atherosclerosis, Central South University;

3. Modern Cardiovascular Disease Clinical Technology Research Center of Hunan Province;

4. Cardiovascular Disease Research Center of Hunan Province; Changsha, Hunan 410011, PR China.

5. Department of Emergency Medicine, Second Xiangya Hospital, Central South University, Changsha, China;

6. Emergency Medicine and Difficult Diseases Institute, Second Xiangya Hospital, Central South University, Changsha, China;

Jin Xu and Peiliu Qu contributed equally to the writing of this article.

* Address correspondence to this author at: \#139 Middle Renmin Road, Changsha, Hunan 410011, PR China. Fax +86-073185295407; E-mail: feliuling@csu.edu.cn. 


\section{Abstract}

2 Background: Hypertension(HBP) is usually accompanied by hypertriglyceridemia that represents the increased triglyceride-rich lipoproteins(TRLs) and their cholesterol content(i.e. remnant cholesterol, RC). According to the European Atherosclerosis Society(EAS), high $\mathrm{RC}(\mathrm{HRC})$ is defined as fasting $\mathrm{RC} \geq 0.8 \mathrm{mmol} / \mathrm{L}$ or/and postprandial $\mathrm{RC} \geq 0.9 \mathrm{mmol} / \mathrm{L}$. However, little was known about postprandial change in RC level after a daily meal in Chinese patients with HBP.

Methods: One hundred and thirty-five subjects, including 90 hypertensive patients(HBP group) and 45 non-HBP controls(CON group), were recruited in this study. Serum levels of blood lipids, including calculated RC, were explored at 0,2 , and $4 \mathrm{~h}$ after a daily breakfast. Receiver operating characteristic(ROC) curve analysis was used to determine the cut-off point of postprandial HRC.

Results: TG and RC levels increased significantly after a daily meal in two groups $(P$ $<0.05)$. However, postprandial RC level was significantly higher in $\operatorname{HBP} \operatorname{group}(P<$ 0.05). ROC curve analysis showed that the optimal cut-off point for RC after a daily meal to predict HRC in corresponding to fasting RC $0.8 \mathrm{mmol} / \mathrm{L}$ was $0.91 \mathrm{mmol} / \mathrm{L}$, which was very close to that recommended by the EAS, i.e. $0.9 \mathrm{mmol} / \mathrm{L}$. Fasting HRC was found in $31.1 \%$ hypertensive patients but not in the controls. According to the postprandial cut-off point, postprandial HRC was found in about half of hypertensive patients and about one third of controls.

Conclusion: Postprandial RC level increased significantly after a daily meal and hypertensive patients had higher percentage of HRC. More importantly, the detection of postprandial lipids is helpful to find HRC.

Keywords: Hypertension; postprandial; remnant cholesterol; cut-off point 
1 Change in postprandial level of remnant cholesterol after a daily breakfast in Chinese patients with hypertension

\section{1. Background}

As important atherogenic risk factors, hypertension(HBP) and hyperlipidemia usually coexist [1]. Evidence showed that hypertriglyceridemia and visceral obesity predicted the prevalence of hypertension in the Chinese population[2, 3]. Hypertriglyceridemia represents the increased number of triglyceride-rich lipoproteins(TRLs) and their remnant lipoproteins(RLPs) in the circulation[4, 5]. Compared with nascent TRLs, RLPs with smaller diameter contains more cholesterol[6]. The atherosclerotic effect of RLPs is no less than that of low-density lipoprotein(LDL)[6]. The content of cholesterol within RLPs is termed as remnant cholesterol(RC). It has been demonstrated that the elevation of RC level can predict the risk of coronary heart disease, just like the increased level of low-density lipoprotein cholesterol(LDL-C)[7-9]. Thus, it is essential to detect RC level in hypertensive patients to assess cardiovascular risk entirely.

$\mathrm{RC}$ level can be calculated as total cholesterol(TC) minus LDL-C minus high-density lipoprotein cholesterol(HDL-C), using fasting or postprandial lipid profiles[10]. It has been known the fasting RC levels in the general population should not exceed 0.8 mmol/L[11-13]. Since 2016, postprandial detection of blood lipids has been recommended in the clinical practice[10]. According to the European joint consensus statement from the European Atherosclerosis Society(EAS), postprandial RC level after a daily meal in the subjects with fasting $\mathrm{RC}<0.8 \mathrm{mmol} / \mathrm{L}$ should not exceed 0.9 $\mathrm{mmol} / \mathrm{L}[10]$. However, the postprandial cut-off point of $\mathrm{RC}$ in corresponding to fasting $\mathrm{RC} 0.8 \mathrm{mmol} / \mathrm{L}$ in the Chinese population is still unclear. In order to conveniently identify the elevation of RC in the postprandial state in Chinese patients with hypertension, we compared the changes in blood lipids between hypertensive patients and their controls after a daily meal in this investigation, and further analyze the optimal postprandial cut-off point of RC in the Chinese individuals after a daily meal in corresponding to fasting $\mathrm{RC} 0.8 \mathrm{mmol} / \mathrm{L}$. 


\section{Methods}

\subsection{Study Subjects}

One hundred and thirty-five subjects, including 90 documented hypertension patients(HBP group) and 45 non-HBP controls(CON group), were recruited in this study in the Department of Cardiovascular Medicine of the Second Xiangya Hospital, Central South University. Hypertension was defined as a history of systolic blood pressure(SBP) values $\geq 140 \mathrm{mmHg}$ and/or diastolic blood pressure(DBP) values $\geq 90$ $\mathrm{mmHg}$ for at least 3 days[14, 15]. Contemporaneous controls who had no clinical history and manifestation of hypertension were classified into CON group.

All subjects were invited to filled out a questionnaire on medical history and use of medication before participant. Patients with secondary hypertension were excluded. No subjects had a history of diabetes, thyroid diseases, liver and kidney diseases, autoimmune disease, cancer or other severe medical illnesses, and no one took oral hypoglycaemic or hypolipidemic agents. This study was approved by the Ethics Committee of the Second Xiangya Hospital of Central South University and informed consent was gained from all participants.

\subsection{Specimen collection}

After at least 12 hours of overnight fasting, venous blood samples were collected in all subjects before(i.e. $0 \mathrm{~h}$ ) and at $2 \mathrm{~h}, 4 \mathrm{~h}$ after a breakfast based on their daily habits. All subjects were requested to finish the meal in 15 minutes. During the 4-hour test, subjects were allowed to drink only water and prohibited to smoke, drink wine or eat any food. Strenuous exercises were not recommended, and only slow walking was allowed.

\subsection{Laboratory Assays}

All blood samples were centrifuged at $4{ }^{\circ} \mathrm{C} 3000 \mathrm{rpm}$ for $15 \mathrm{~min}$. Serum levels of TC and triglyceride(TG) were measured by automated enzymatic assays, and that of HDL-C were measured by a commercially available direct method, on a HITACHI 7170A analyzer(Instrument Hitachi Ltd., Tokyo, Japan) by a laboratory technician who had no idea of this study[9, 16]. LDL-C level was calculated using the 
1 Friedewald formula: LDL-C $=$ TC $-(\mathrm{HDL}-\mathrm{C})-(\mathrm{TG} / 2.2)$ when TG was $<4.5 \mathrm{mmol} / \mathrm{L}$,

2 otherwise it was directly measured by chemical masking method. RC level was

3 estimated by the following formula, $\mathrm{RC}=\mathrm{TC}-(\mathrm{HDL}-\mathrm{C})-(\mathrm{LDL}-\mathrm{C})$. Non-HDL-C $=\mathrm{TC}$

4 -(HDL-C)[10] .

5 2.4. Statistical analysis

6 Quantitative variables were expressed as mean \pm standard deviation(SD) unless were

7 specifically explained, and qualitative variables were expressed as numbers and

8 percentages. Differences between the intra- and intergroup means were analyzed by unpaired $t$-test or one-way analysis of variance. Categorical variables were compared using chi-squared test. The area under the curve(AUC) was estimated by trapezoid method. The optimal cut-off point for postprandial RC level was determined using receiver operating characteristic(ROC) curve analysis[9]. All statistical analyses were performed with SPSS version 25.0. All $P$ values were 2 -tailed, and $P<0.05$ was considered statistically significant.

\section{Result}

\subsection{Clinical characteristics and fasting blood lipids of two groups}

There was no significant difference in age, gender, body mass index(BMI), diastolic blood pressure, heart rate and percentage of overweight or smoking between two groups. Systolic blood pressure was significantly higher in HBP group. Moreover, levels of fasting TC, TG, non-HDL-C and RC were significantly higher in HBP $\operatorname{group}(P<0.05$, Table 1), while fasting levels of HDL-C and LDL-C were similar between two groups.

\subsection{Postprandial changes in serum levels of blood lipids in two groups}

After a daily breakfast, postprandial reduction in levels of TC, HDL-C and LDL-C were slight but significant(Fig.1A-C). Postprandial non-HDL-C level significantly decreased in HBP group $(P<0.05)$ but not in CON group(Fig 1D). Postprandial levels of TG and RC increased tremendously in two groups $(P<0.05$, Fig $1 \mathrm{E} \& \mathrm{~F})$.

Levels of TC, TG, non-HDL-C and RC after a daily meal in HBP group were significantly higher than those in $\mathrm{CON}$ group, while there was no significant 
1 difference in postprandial levels of HDL-C and LDL-C between two groups just as

2 what they were presented in the fasting $\operatorname{state}(P<0.05$, Fig. 1A-F $)$.

3 AUCs of TC, TG, non-HDL-C and RC in HBP group were significantly higher than 4 those in CON group $(P<0.05)$, however, AUCs of HDL-C and LDL-C were similar in 5 two groups(Fig 1G).

\subsection{The contribution of blood lipids to hypertension}

To determine the contribution of blood lipids to hypertension, logistic regression analysis was performed. Among all lipid profiles at fasting state, only fasting RC level was independently contributed to the occurrence of hypertension(OR, 68.869; 95\% CI, 8.533-554.560; $P<0.001)$.

For the close relationship between fasting and postprandial $\mathrm{RC}$ levels at $2 \mathrm{~h}(\mathrm{r}=0.73$, $P<0.001$ ) or $4 \mathrm{~h}(\mathrm{r}=0.64, P<0.001)$, the contribution of postprandial $\mathrm{RC}$ levels to hypertension was also evaluated by regression analysis. In addition to fasting RC level, postprandial $\mathrm{RC}$ level at $4 \mathrm{~h}$ was independently contributed to the occurrence of hypertension(OR, 2.435; 95\% CI, 1.044-5.675; $P<0.001$ ).

\subsection{Determination of the postprandial optimal cut-off point corresponding to} fasting high RC(HRC).

Considering postprandial RC level reached peak value at $4 \mathrm{~h}$ after a daily breakfast, ROC analysis was performed and Youden's index was calculated to determine the postprandial optimal cut-off point at $4 \mathrm{~h}$. The optimal cut-off point for $\mathrm{RC}$ at $4 \mathrm{~h}$ to predict $\mathrm{HRC}$ in relation to fasting RC $0.8 \mathrm{mmol} / \mathrm{L}$ was $0.9095 \mathrm{mmol} / \mathrm{L}$ (sensitivity $82.1 \%$, specificity $70.1 \%$, and AUC $0.806 ; P<0.001$, Fig. $2 \mathrm{~A}$ ), which was closely to the optimal cut-off point, $0.9 \mathrm{mmol} / \mathrm{L}$, after a daily meal recommended by the EAS expert consensus on the detection of postprandial blood lipids. Moreover, when RC levels at $2 \mathrm{~h}$ and $4 \mathrm{~h}$ were pooled together, ROC analysis also showed $0.9095 \mathrm{mmol} / \mathrm{L}$ as the optimal cut-off point after a daily meal in relation to fasting RC 0.8 mmol/L(sensitivity $83.9 \%$, specificity $71.0 \%$, and AUC 0.832; $P<0.001$, Fig. $2 \mathrm{~B}$ ).

\subsection{Comparisons of the percentages of postprandial HRC between two groups.}

According to the optimal cut-off point after a daily meal recommended by the EAS expert consensus[10], fasting HRC(fHRC) was found in $31.1 \%$ subjects in HBP group, 
1 however, the percentages of postprandial $\mathrm{HRC}(\mathrm{pHRC})$ significantly increased to

$244.4 \%$ at $2 \mathrm{~h}$ and $47.8 \%$ at $4 \mathrm{~h}$, respectively $(P<0.05)$. When RC level was detected at

3 both fasting and postprandial states at $2 \mathrm{~h}$ or $4 \mathrm{~h}$ in the same subjects, the percentages

4 of HRC significantly increased to $48.9 \%$ or $53.3 \%(P<0.05$, Fig $3 \mathrm{~A} \& \mathrm{~B})$.

5 Although the fasting RC levels of the subjects in CON group were $<0.8 \mathrm{mmol} / \mathrm{L}$,

$6 \mathrm{pHRC}$ was found in $35.6 \%$ subjects at $2 \mathrm{~h}$ and in $33.3 \%$ ones at $4 \mathrm{~h}$, respectively $(P<$

$7 \quad 0.05$, Fig 3A\&B).

\section{Discussion}

In this study, the optimal cut-off point of postprandial RC level after a daily meal in corresponding to fasting $\mathrm{RC}$ level $0.8 \mathrm{mmol} / \mathrm{L}$ was firstly determined in Chinese subjects. Interestingly, it was close to that recommended by the EAS expert consensus[10]. Moreover, higher RC level as well as higher proportion of HRC were found in HBP group in both fasting and postprandial states, suggesting that hypertension patients could be at greater cardiovascular risk due to abnormal TRL metabolism, in addition to hypertension.

RC level can be accurately detected through several expensive and complex methods, including ultracentrifugation, nuclear magnetic resonance, immune separation and so on[17-19]. However, those kinds of accurate detection are very difficult to be widely used in the primary hospitals. The formula method recommended by the EAS expert consensus gives doctors the opportunity to estimate the RC levels of subjects at no additional cost[10]. Elevation of RC level indicated the excessive overproduction of nascent TRLs and/or delayed removal of RLPs in patients with hypertension. And this situation persisted in the postprandial state. Similar conditions were also found in TG level. Those results suggested that there is a close relationship between hypertension and abnormal metabolism of TRLs/RLPs.

Hypertriglyceridemia could be involved in the occurrence of hypertension through an aldosterone-dependent pathway[20-22]. It has been showed that the expression and secretion of aldosterone in adrenal cells were influenced by TRLs and their oxidized particles[23, 24]. Local synthesis of aldosterone in extra-adrenal tissues, including 
1 adipose tissue, was also reported[22, 25]. Positive correlation between plasma

2 aldosterone levels and body mass index supported a relationship between adipose tissue and the production and secretion of aldosterone[26]. In this study, HBP group seemed to have higher BMI and more subjects with overweight, indicating that obesity is an important link between hypertriglyceridemia and hypertension. TRLs and RLPs take part in the occurrence obesity through inducing adipogenic differentiation of preadipocytes[27] and hypertrophy of adipocytes[28]. Adipokines, such as leptin and adiponectin, released by the hypertrophic adipocytes may affect aldosterone secretion of adrenal gland in a paracrine manner[29]. It indicated that TRLs and RLPs promoted the release of aldosterone, directly and indirectly, which plays an important role in the pathogenesis of hypertension.

A previous study had shown that high fasting RC level was associated with the development of hypertension after 10 years in subjects who had normal blood pressure at baseline[30]. Different from nascent TRLs with too large particles, RLPs with smaller diameter had a more definite effect on vascular cells[6]. Endothelial dysfunction is one of the key mechanisms of hypertension[27, 31]. On the one hand, postprandially increased RLPs can directly impair arterial vasodilation, which has been demonstrated in the patients after a high-fat meal and the separated vascular ring incubated with RLPs[32, 33]. On the other hand, RLPs induce endothelial inflammaion, increase the production of vasoconstrictor and the oxidative stress of endothlial cells[34]. More importantly, RLPs can penetrate into the subendothelial area and then directly impair the function of vascular smooth muscles[35, 36]. Above evidence supports that RLPs impair endothelium depentdent- and independent-vasodilation, which could partly explain the contribution of RC level to the occurrence of hypertension.

It is well known that people spend most of the day in the postprandial state. Moreover, a considerable number of subjects are difficult to maintain fasting or unwilling to be fasting during the visit in the medical services. Thus, some patients with high TG or $\mathrm{RC}$ level may be missed if the detection of blood lipids only can be carried out in the fasting state. According to the EAS expert consensus and the statement from the 
1 American Heart Association(AHA), the postprandial TG level in an individual with 2 fasting TG $<1.7 \mathrm{mmol} / \mathrm{L}$ should not raise above $2.0 \mathrm{mmol} / \mathrm{L}$ and $2.26 \mathrm{mmol} / \mathrm{L}$, 3 respectively, after consuming a daily meal[10,13]. Recently, we determined a cut-off 4 point for postprandial TG level $2.02 \mathrm{mmol} / \mathrm{L}$ at $4 \mathrm{~h}$ after a daily breakfast in corresponding to fasting TG level $1.7 \mathrm{mmol} / \mathrm{L}$ in Chinese subjects[37], which is close to the cut-off point for postprandial TG level $2.0 \mathrm{mmol} / \mathrm{L}$ recommended by the EAS expert consensus[10]. However, expect for the EAS expert consensus, there was no recommendation about fasting and postprandial $\mathrm{RC}$ levels in the United States or China.

In this study, the cut-off point of postprandial RC level in corresponding to fasting $\mathrm{RC}$ level $0.8 \mathrm{mmol} / \mathrm{L}$ was about $0.91 \mathrm{mmol} / \mathrm{L}$ after a daily meal in Chinese subjects, which was quite near to that recommended by the EAS expert consensus, i.e. 0.9 $\mathrm{mmol} / \mathrm{L}[10]$. It suggests that Chinese subjects may share similar cut-off points with the Europeans after a daily meal. When postprandial RC $0.9 \mathrm{mmol} / \mathrm{L}$ was used to evaluate the percentage of postprandial HRC in each group, postprandial HRC was found in more patients in HBP group and about one third ones in CON group, although their fasting RC levels were $<0.8 \mathrm{mmol} / \mathrm{L}$. The postprandial increase in HRC in two groups could be not only associated with their own habitual breakfasts, but also with the existence of some subjects with overweight and smoking in each group. Decreased hydrolysis of TRLs was reported in smokers and patients with obesity[38-40]. If both fasting and postprandial blood lipids can be detected in a certain person, the diagnostic rate of HRC will be further improved, although it is not feasible in the real world. Certainly, the detection of postprandial RC level can find more patients with HRC, and is worth carrying out in clinical practice.

This study is associated with several limitations. Firstly, the number of cases in this study was relatively small when compared with other similar studies[41]. Secondly, some subjects had breakfasts in the hospital canteen, which could be different from their usual diets at home. Thirdly, both LDL-C and RC were calculated by Friedewald formula, which may cause deviation with those directly measured or calculated by other formulas[42]. 
1 In conclusion, postprandial RC level increased significantly after a daily meal and

2 patients with hypertension had significantly higher percentage of HRC than the

3 controls. More importantly, the postprandial detection of blood lipids is helpful to find

4 HRC.

5

\section{Declarations}

7 Abbreviations

8 AHA: American Heart Association; AUC: Area under the curve; BMI: body mass

9 index; CON: Non-HBP controls; DBP: diastolic blood pressure; EAS: European

10 Atherosclerosis Society; HBP: hypertension; HDL-C: High-density lipoprotein

11 cholesterol; HRC: High remnant cholesterol; LDL: Low-density lipoprotein; LDL-C:

12 Low-density lipoprotein cholesterol; Non-HDL-C: Nonhigh-density lipoprotein

13 cholesterol; RC: Remnant cholesterol; ROC curve: Receiver operating characteristic;

14 SBP:systolic blood pressure; TC: Total cholesterol; TG: Triglyceride; TRLs:

15 Triglyceride-rich lipoproteins

16 Funding

17 The study was supported by National Natural Science Foundation of China [grant

18 numbers 81270956,81470577$]$.

\section{Authors' contributions}

20 All authors have accepted responsibility for the entire content of this manuscript and

21 approved its submission.

\section{Competing Interests}

23 The authors declare that they have no competing interests.

\section{Availability of data and materials}

The datasets analyzed during the current study are available from the corresponding author on reasonable request. 


\section{Ethics approval and consent to participate}

2 The study was approved by the Ethics Committee of the Second Xiangya Hospital of

3 Central South University and informed consent was gained from all participants.

\section{Reference}

1. Sander, G. and T. Giles, Hypertension and lipids: Lipid factors in the hypertension syndrome.

2. Feng, R.N., et al., BMI is strongly associated with hypertension, and waist circumference is

3. Han, M., et al., Chinese visceral adiposity index, a novel indicator of visceral obesity for

4. Kolovou, G. and T.C. Ooi, Postprandial lipaemia and vascular disease. Curr Opin Cardiol, 2013. 28(4): p. 446-51.

5. Fernandez-Arroyo, S., et al., Effect of Vitamin D3 on the Postprandial Lipid Profile in Obese Patients: A Non-Targeted Lipidomics Study. Nutrients, 2019. 11(5).

6. Blackburn, P., et al., Postprandial hyperlipidemia: another correlate of the "hypertriglyceridemic waist" phenotype in men. Atherosclerosis, 2003. 171(2): p. 327-36.

7. McNamara, J.R., et al., Remnant-like particle(RLP) cholesterol is an independent cardiovascular disease risk factor in women: results from the Framingham Heart Study. Atherosclerosis, 2001. 154(1): p. 229-36.

8. Peng, J., et al., Hypertriglyceridemia and atherosclerosis. Lipids Health Dis, 2017. 16(1): p. 233.

9. $\mathrm{Xu}, \mathrm{J}$,, et al., Determination of optimal cut-off points after a high-fat meal corresponding to fasting elevations of triglyceride and remnant cholesterol in Chinese subjects. Lipids Health Dis, 2019. 18(1): p. 206.

10. Nordestgaard, B.G., et al., Fasting is not routinely required for determination of a lipid profile: clinical and laboratory implications including flagging at desirable concentration cut-points-a joint consensus statement from the European Atherosclerosis Society and European Federation of Clinical Chemistry and Laboratory Medicine. Eur Heart J, 2016. 37(25): p. 1944-58.

11. Hu, D.Y., [New guidelines and evidence for the prevention and treatment of dyslipidemia and atherosclerotic cardiovascular disease in China]. Zhonghua Xin Xue Guan Bing Za Zhi, 2016. 44(10): p. 826-827.

12. Catapano, A.L., et al., 2016 ESC/EAS Guidelines for the Management of Dyslipidaemias. Eur Heart J, 2016. 37(39): p. 2999-3058.

13. Ray, K.K., et al., The ACC/AHA 2013 guideline on the treatment of blood cholesterol to reduce atherosclerotic cardiovascular disease risk in adults: the good the bad and the uncertain: a comparison with ESC/EAS guidelines for the management of dyslipidaemias 2011. Eur Heart J, 2014. 35(15): p. 960-8.

14. Bureau of Disease, P., et al., [National guideline for hypertension management in 
China(2019)]. Zhonghua Xin Xue Guan Bing Za Zhi, 2020. 48(1): p. 10-46.

15. Whelton, P.K., et al., 2017 ACC/AHA/AAPA/ABC/ACPM/AGS/APhA/ASH/ASPC/NMA/PCNA Guideline for the Prevention, Detection, Evaluation, and Management of High Blood Pressure in Adults: A Report of the American College of Cardiology/American Heart Association Task Force on Clinical Practice Guidelines. J Am Coll Cardiol, 2018. 71(19): p. e127-e248.

16. Xiang, Q.Y., et al., Comparison of remnant cholesterol levels estimated by calculated and measured LDL-C levels in Chinese patients with coronary heart disease. Clin Chim Acta, 2020. 500: p. 75-80.

17. Okazaki, M., et al., Relation between RLP-triglyceride to RLP-cholesterol ratio and particle size distribution in RLP-cholesterol profiles by HPLC. Clin Chim Acta, 2000. 296(1-2): p. 135-49.

18. Leary, E.T., et al., Evaluation of an immunoseparation method for quantitative measurement of remnant-like particle-cholesterol in serum and plasma. Clin Chem, 1998. 44(12): p. 2490-8.

19. Nakada, Y., et al., Increased remnant lipoprotein in patients with coronary artery disease--evaluation utilizing a newly developed remnant assay, remnant lipoproteins cholesterol homogenous assay (RemL-C). J Atheroscler Thromb, 2007. 14(2): p. 56-64.

20. Armitage, J.A., et al., Rapid onset of renal sympathetic nerve activation in rabbits fed a high-fat diet. Hypertension, 2012. 60(1): p. 163-71.

21. Vogt, B., M. Bochud, and M. Burnier, The association of aldosterone with obesity-related hypertension and the metabolic syndrome. Semin Nephrol, 2007. 27(5): p. 529-37.

22. Briones, A.M., et al., Adipocytes produce aldosterone through calcineurin-dependent signaling pathways: implications in diabetes mellitus-associated obesity and vascular dysfunction. Hypertension, 2012. 59(5): p. 1069-78.

23. Tsai, Y.Y., et al., Phospholipase D activity underlies very-low-density lipoprotein(VLDL)-induced aldosterone production in adrenal glomerulosa cells. Endocrinology, 2014. 155(9): p. 3550-60.

24. Tsai, Y.Y., W.E. Rainey, and W.B. Bollag, Very low-density lipoprotein(VLDL)-induced signals mediating aldosterone production. J Endocrinol, 2017. 232(2): p. R115-R129.

25. Nguyen Dinh Cat, A., et al., Adipocyte-derived factors regulate vascular smooth muscle cells through mineralocorticoid and glucocorticoid receptors. Hypertension, 2011. 58(3): p. 479-88.

26. Rossi, G.P., et al., Body mass index predicts plasma aldosterone concentrations in overweight-obese primary hypertensive patients. J Clin Endocrinol Metab, 2008. 93(7): p. 2566-71.

27. Zhang, M., et al., Indispensable role of lipoprotein bound-ApoE in adipogenesis and endocytosis induced by postprandial TRL. Biochem Biophys Res Commun, 2017. 493(1): p. 298-305.

28. Nguyen, A., et al., Very low density lipoprotein receptor(VLDLR) expression is a determinant factor in adipose tissue inflammation and adipocyte-macrophage interaction. J Biol Chem, 2014. 289(3): p. 1688-703.

29. Dinh Cat, A.N., et al., Adipocytes, aldosterone and obesity-related hypertension. J Mol Endocrinol, 2016. 57(1): p. F7-F21.

30. Kasahara, A., et al., High level of plasma remnant-like particle cholesterol may predispose to development of hypertension in normotensive subjects. Am J Hypertens, 2013. 26(6): p. 793-8.

31. Kalra, J., et al., PKR inhibitor imoxin prevents hypertension, endothelial dysfunction and cardiac and vascular remodelling in L-NAME-treated rats. Life Sci, 2020. 262: p. 118436. 
32. Funada, J., et al., Postprandial elevation of remnant lipoprotein leads to endothelial

33. Doi, H., et al., Membrane active lipids in remnant lipoproteins cause impairment of

40. Mekki, N., et al., Influence of obesity and body fat distribution on postprandial lipemia and triglyceride-rich lipoproteins in adult women. J Clin Endocrinol Metab, 1999. 84(1): p. 184-91.

41. Hwang, Y.C., et al., Optimal range of triglyceride values to estimate serum low density lipoprotein cholesterol concentration in Korean adults: the Korea National Health and Nutrition Examination Survey, 2009. J Korean Med Sci, 2012. 27(12): p. 1530-5.

42. Lin, Q.Z., et al., Comparison of non-fasting LDL-C levels calculated by Friedewald formula with those directly measured in Chinese patients with coronary heart disease after a daily breakfast. Clin Chim Acta, 2019. 495: p. 399-405. 

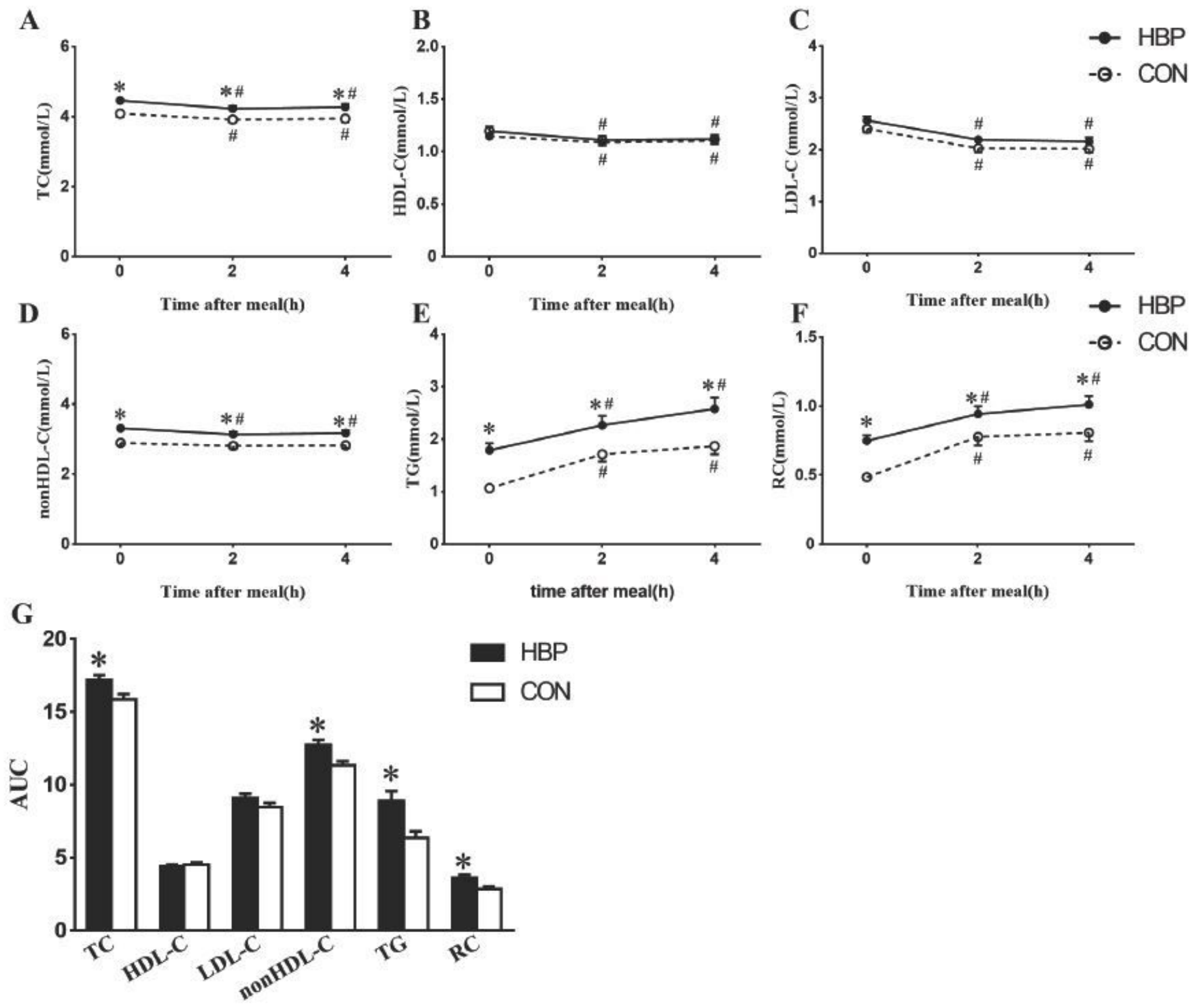

\section{Figure 1}

Changes in serum levels of blood lipids after a daily meal in two groups. (A-F) Postprandial changes in serum levels of TC, HDL-C, non-HDL-C, LDL-C, TG, and RC after a daily meal in HBP group (solid line) and CON group (dotted line). The bar represent standard error of the mean. (G) Comparison of AUC of blood lipids after a daily meal between two groups. * $\mathrm{P}<0.05$ when compared with CON group at the same time point. \# $\mathrm{P}<0.05$ when compared with fasting level in the same group. 
$\mathbf{A}$

\section{ROC estimates for RC4h}

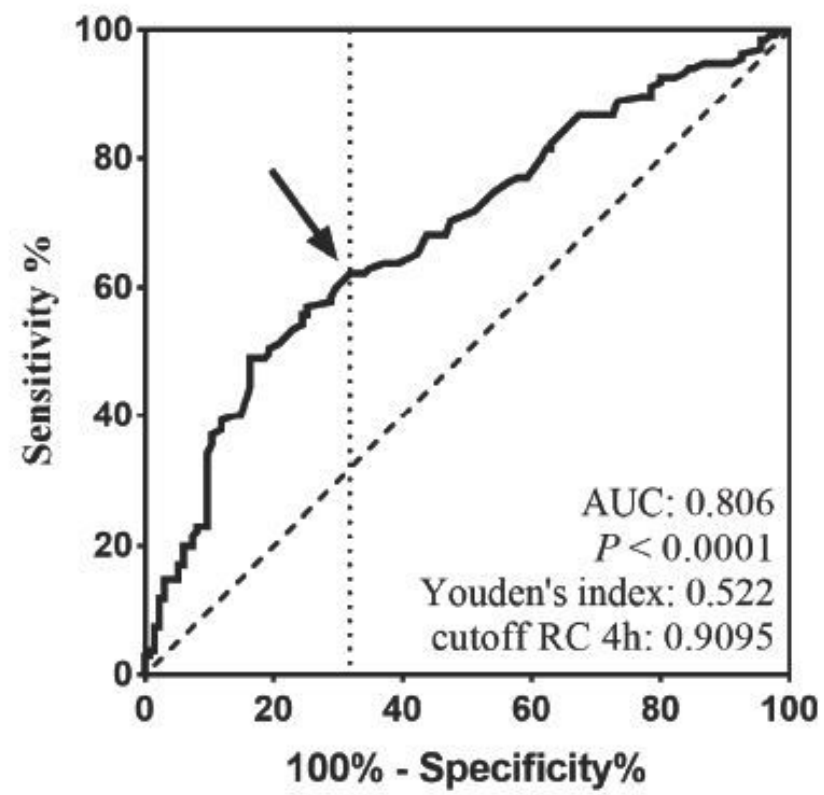

B
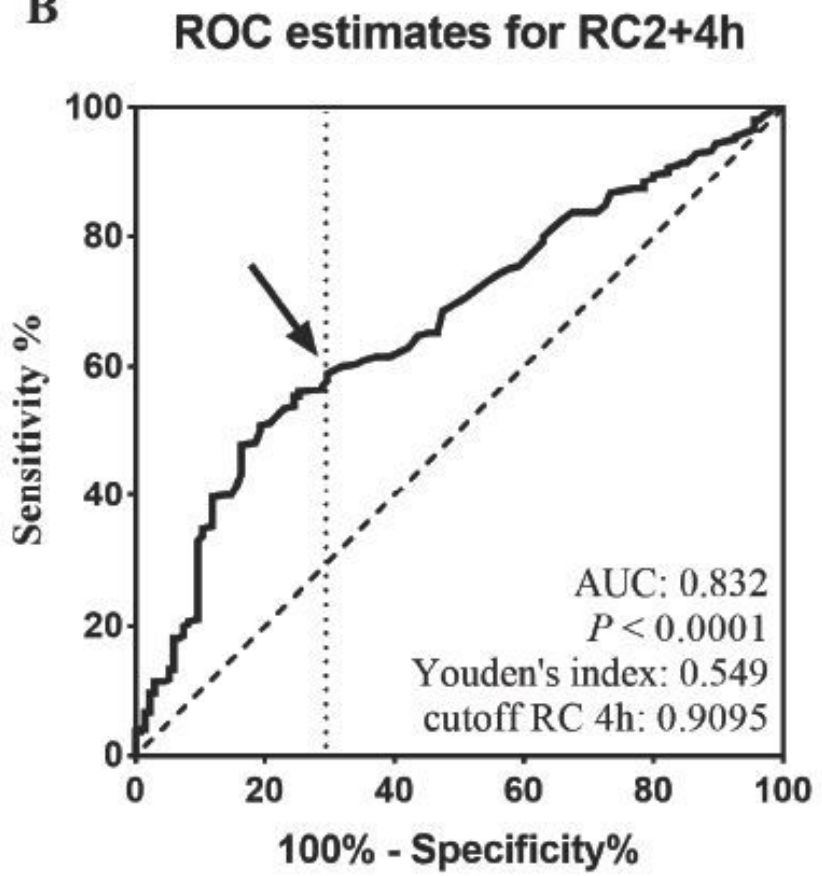

Figure 2

Determination of the postprandial optimal cut-off point corresponding to fasting high RC.(A, B) ROC analysis and Youden's index determined a cut-off point for postprandial HRC at $4 \mathrm{~h}(\mathrm{pRC} 4 \mathrm{~h}$ ) or at both $2 \mathrm{~h}$ and $4 \mathrm{~h}(\mathrm{pRC} 2+4 \mathrm{~h})$ after a daily meal, the cut-off point was indicated by the solid arrow.
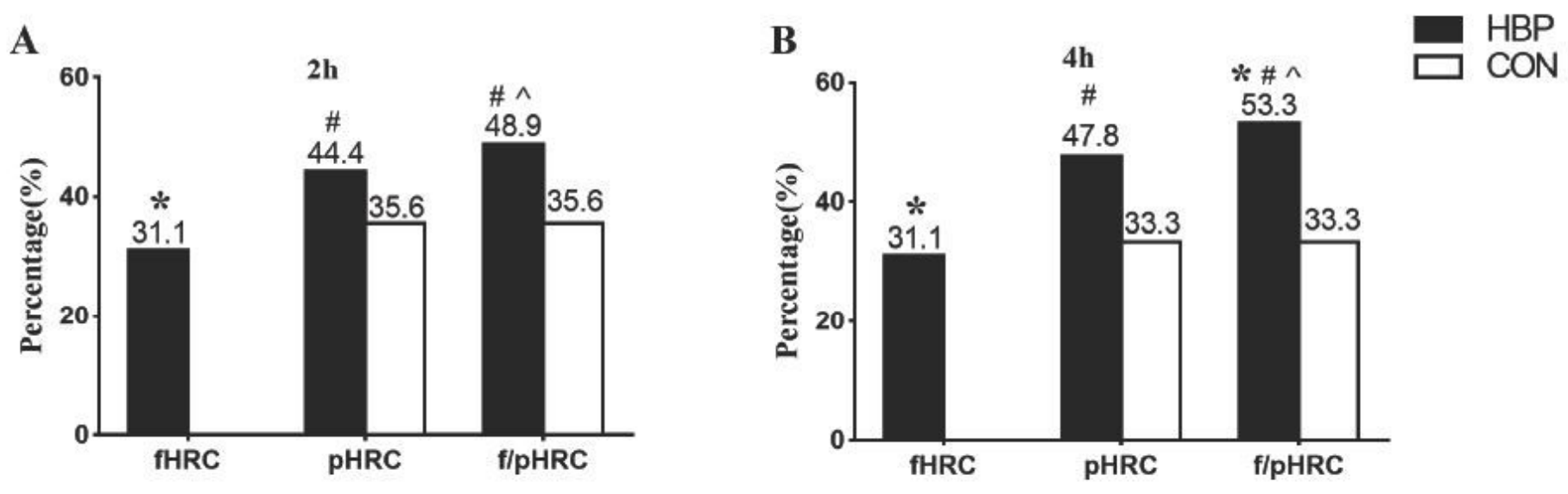

Figure 3

Comparisons of the percentages of HRC between two groups at different states. (A, B) Comparisons of the percentages of fasting HRC only(fasting $R C \geq 0.8 \mathrm{mmol} / \mathrm{L}$, $\mathrm{HHRC}$ ), postprandial HRC only(postprandial $\mathrm{RC} \geq 0.9 \mathrm{mmol} / \mathrm{L}, \mathrm{pHRC}$ ), either fasting or postprandial $\mathrm{HRC}($ fasting $\mathrm{RC} \geq 0.8 \mathrm{mmol} / \mathrm{L}$ or postprandial 
$\mathrm{RC} \geq 0.9 \mathrm{mmol} / \mathrm{L}, \mathrm{f} / \mathrm{pHRC}$ ) at $2 \mathrm{~h}$ or $4 \mathrm{~h}$ after a daily breakfast. ${ }^{*}<0.05$ when compared with CON group. \# $\mathrm{P}<0.05$ when compared with the percentage at fasting state in HBP group. $\otimes \mathrm{P}<0.05$ when compared with the percentage of $\mathrm{pHRC}$ in HBP group.

\section{Supplementary Files}

This is a list of supplementary files associated with this preprint. Click to download.

- Table1.docx 